Jul 1st, 12:00 AM

\title{
Risk Assessment for Sustainable Food Security in China According to Integrated Food Security - Taking Dongting Lake Area for Example
}

Xiaoxing Qi

Liming Liu

Follow this and additional works at: https://scholarsarchive.byu.edu/iemssconference

Qi, Xiaoxing and Liu, Liming, "Risk Assessment for Sustainable Food Security in China According to Integrated Food Security_Taking Dongting Lake Area for Example" (2012). International Congress on Environmental Modelling and Software. 274. https://scholarsarchive.byu.edu/iemssconference/2012/Stream-B/274

This Event is brought to you for free and open access by the Civil and Environmental Engineering at BYU ScholarsArchive. It has been accepted for inclusion in International Congress on Environmental Modelling and Software by an authorized administrator of BYU ScholarsArchive. For more information, please contact scholarsarchive@byu.edu, ellen_amatangelo@byu.edu. 


\title{
Risk Assessment for Sustainable Food Security in China According to Integrated Food Security-Taking Dongting Lake Area for Example
}

\author{
Xiaoxing $\mathbf{Q}^{\mathrm{a}}$, Liming Liu $^{\mathrm{a}}$ \\ ${ }^{a}$ College of Resources \& Environment, China Agricultural University, Beijing \\ 100193, China \\ uniop@126.com
}

\begin{abstract}
The risk assessment of regional sustainable food security is one of the most important issues, and is closely related to regional sustainable development and maintaining social stability. This paper mainly focuses on the characteristics of sustainable food security problems in the major grain-producing areas in China. We establish an index system based on land resources and eco-environmental conditions and apply a dynamic assessment method based on status assessments and trend analysis models to overcome the shortcomings of the static evaluation method. Using fuzzy mathematics, the risks are categorized into four grades: negligible risk, low risk, medium risk and high risk. A case study was conducted in one of China's major grain-producing areas: Dongting Lake area. The results predict that the status of the sustainable food security in the Dongting Lake area is unsatisfactory for the foreseeable future. Therefore, appropriate policies and measures should be put forward to improve it.
\end{abstract}

Keywords: Food security; Risk assessment; Regional scale; Dongting Lake area.

\section{INTRODUCTION}

Sustainable food security is a complex concept containing three interactional aspects: the sustainability of the food production, the sustainability of the local retail market for food and the sustainability of resources and the environment. However, thus far, few studies of sustainable food security focus on the food supply chain, food systems and firm-level economic performance [llbery and Maye 2005]. Resources and the environment provide the basis for the three aspects because they are the direct source of the food security. Furthermore, the results of sustainable food security research in terms of resources and the environment (in this paper, resources and the environment mainly refer to regional land resources and eco-environmental conditions which could be influenced by human activities, respectively) can provide relevant information for agricultural insurance systems and policy makers in land management departments and the agricultural sector. Thus, it is very relevant to study sustainable food security by focusing on the sustainability of resources and the environment.

Because of rapid population growth, severe soil and water loss, serious environmental pollution and degradation of land resources, China has been challenged by food security. The view that "Chinese food security is based on resources and the environment" has been put forward by many domestic scholars in recent years. Because the relevant studies were normally conducted at the 
national level and their results were neither quantified nor made available to decision makers, there has long been a gap between scientific research and the concrete practice of pursuing food security [Yang and Li 2000; Yanet et al. 2009]. By using a risk assessment method to perform the study at a regional scale, the gap will be bridged when the results can be presented quantitatively and visually [Smith and König 2010].

This paper is driven by the view that there is applied value in studying sustainable food security in terms of risk assessment, from the perspective of resources and the environment, at the regional scale.

\section{MATERIALS AND METHODS}

As sustainable food security risk varies due to different land resources and ecoenvironmental conditions, it is necessary to select a representative research area.

\subsection{Study area and data source}

This paper examines the Dongting Lake area $\left(110^{\circ} 29^{\prime} \sim 114^{\circ} 15^{\prime} \mathrm{E}\right.$ and $26^{\circ} 03^{\prime} \sim$ $30^{\circ} 08^{\prime} \mathrm{N}$ ). It is located in northern Hunan Province and includes 6 cities and 29 counties, which together occupy an area of about $73,500 \mathrm{~km}^{2}$. The area has a humid climate, with a mean annual rainfall of $1305 \mathrm{~mm}$ and a mean annual temperature of $16.8^{\circ} \mathrm{C}$, and has long been a major grain-producing area in China. The raw data were taken from the 1986 to 2008 editions of the Hunan Statistical Yearbook, the Hunan Province Agricultural Statistical Yearbook, the Changsha Statistical Yearbook, the Zhuzhou Statistical Yearbook, the Xiangtan Statistical Yearbook, the Yiyang Statistical Yearbook, the Changde Statistical Yearbook and the Yueyang Statistical Yearbook.

\subsection{Risk assessment}

Because sustainable food security risk is a new concept, there are no universal evaluation standards. However, the core of risk assessment for sustainable food security is, by its very nature, risk assessment. Based on some classical guidelines, methods and principles of risk assessment, this paper proposes a framework for an integrated three-step risk assessment for sustainable food security procedure, i.e., risk identification, risk analysis and risk characterization [Smith and König 2010; Knudsen 2010].

\subsubsection{Risk identification}

The purpose of this step is to discover the causes of sustainable food security risk. Overall, relevant research indicates that there are six traditional causes that can influence food security: disaster risk, income risk, pollution risk, resources risk, yield risk and trade risk [Khan and Hanjra 2009; Wu et al. 2010]. Due to the focus on regional land resources and eco-environmental conditions, this paper does not consider income risk and trade risk. The socio-economic sources that have little direct influence on land resources and eco-environmental conditions are also not considered.

With reference to the above discussion, this paper identifies three sources that could cause sustainable food security risk: resource risk, pollution risk and disaster risk.

\subsubsection{Risk analysis}


Based on the three risk sources identified above, relevant risk factors and concrete evaluation indexes that could be used to evaluate sustainable food security risk are formulated in this step. Then, the weights and the risk thresholds of these indexes are defined.

\subsubsection{Construction of index system}

Based on the resources and environmental situation of the Dongting Lake area and some relevant research, this paper proposes that the three risk sources contain several risk factors, which are as follows [Nie et al. 2010]. For resources risk, population pressure, resource endowment and land degradation are the main risk factors. Pollution risk generally includes agricultural pollution and industrial pollution. While disaster risk includes many risk factors, natural disaster is the only main factor from the historical data of the Dongting Lake area.

Based on the above methods, this paper constructs an index system for risk assessment for sustainable food security in the Dongting Lake area as Fig. 1 shows.

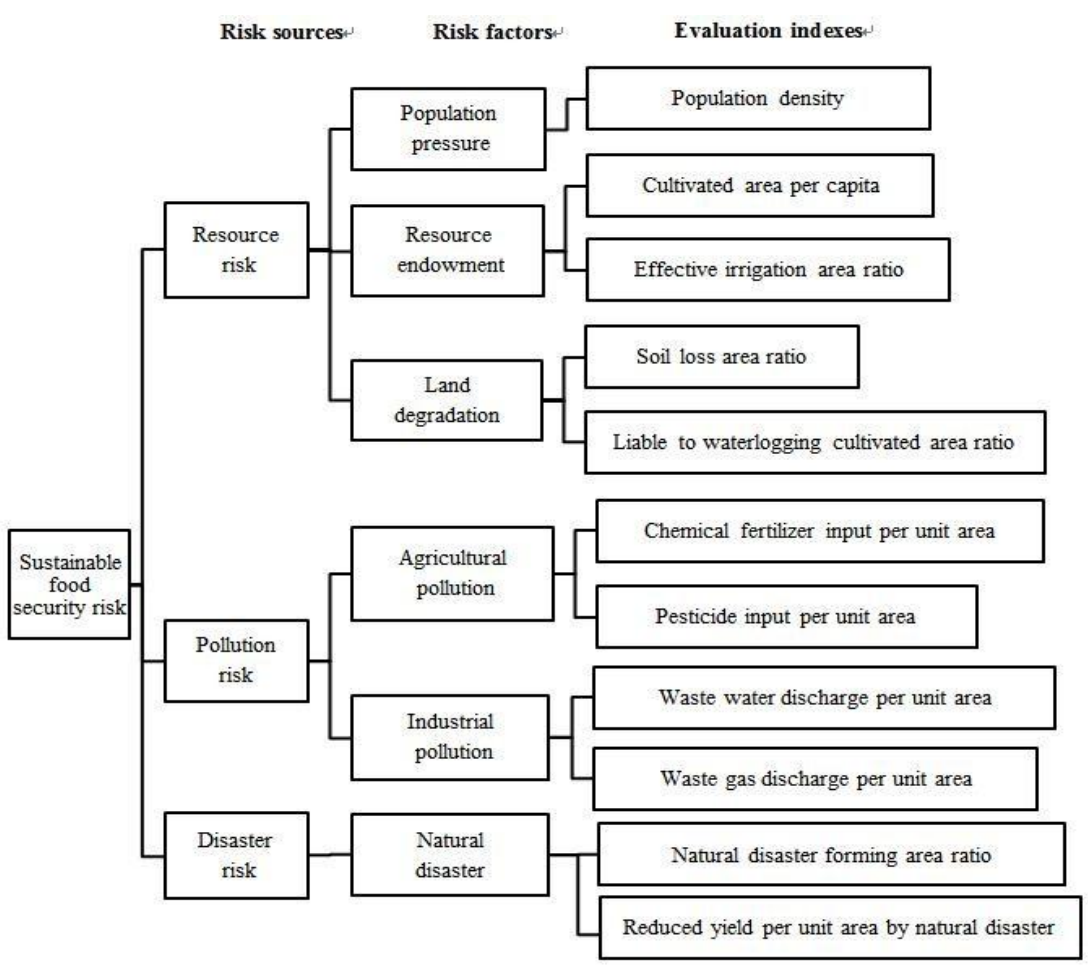

Figure 1. Index system of risk assessment for sustainable food security in Dongting Lake area.

\subsubsection{Weights and risk thresholds of evaluation indexes}

After formulating the evaluation indexes, their weights and risk thresholds need to be defined. This paper uses a principal components analysis (PCA) method to objectively calculate the weights of these indexes because there is little experience with determining them subjectively. After using SPSS Statistics (version 19.0) to make a PCA of the evaluation indexes with a simple calculation, their weights are shown in Table 1 [Wu et al. 2009]. 
The evaluation indexes above can be divided into two types. For some of them, the higher its value, the more security it reflects. These indexes are called "positive indexes." For the remainder, the higher the value, the less security it reflects. These indexes are called "reverse indexes." In this paper, the cultivated area per capita and the effective irrigation area ratio are positive indexes, while all the others are reverse indexes. For positive indexes, if the actual value is higher than the respective risk threshold, there will be no risk. For reverse indexes, it is the opposite.

Table 1. The weights of evaluation indexes of risk assessment for sustainable food security in Dongting Lake area

\begin{tabular}{cc}
\hline Evaluation indexes & Weights \\
\hline Population density & 0.101 \\
Cultivated area per capita & 0.100 \\
Effective irrigation area ratio & 0.087 \\
Soil loss area ratio & 0.095 \\
Liable to waterlogging cultivated area ratio & 0.097 \\
Chemical fertilizer input per unit area & 0.100 \\
Pesticide input per unit area & 0.095 \\
Waste water discharge per unit area & 0.096 \\
Waste gas discharge per unit area & 0.097 \\
Natural disaster forming area ratio & 0.085 \\
Reduced yield per unit area by natural disaster & 0.047 \\
\hline
\end{tabular}

However, the risk thresholds of these evaluation indexes remain at the exploratory phase. This paper takes the average values of these indexes for Hunan Province from 1986 to 2008, rather than the average for all of China, as the risk thresholds. Because maintaining the index averages of the past 20 years would provide a satisfactory status and would be a challenging goal for policy makers.

\subsubsection{Risk characterization}

Although risk index methods are widely used to evaluate eco-environmental conditions in the academic world, they cannot be reflected visually. Based on the risk index method, this paper classifies the risk grades of sustainable food security by using fuzzy mathematics [Verbruggen and Zimmermenu 1999; Shi et al. 2006].

\subsubsection{Evaluation model}

Because the dimensions of risk assessment for sustainable food security evaluation indexes vary, this paper uses a risk index calculating model to compare them.

For a single index, the index calculating models are as follows (function (1) and (2), respectively, for the positive indexes and the reverse indexes):

$$
r_{i}=\left\{\begin{array}{cc}
x_{i} / z_{i}, & \text { if } x_{i}<z_{i} \\
1, & \text { if } x_{i} \geq z_{i}
\end{array} \text { (1) } \quad r_{i}=\left\{\begin{array}{cc}
1, & \text { if } x_{i} \leq z_{i} \\
z_{i} / x_{i}, & \text { if } x_{i}>z_{i}
\end{array}\right.\right.
$$

where $r_{i}$ is the single risk index, $0 \leq r_{i} \leq 1, x i$ and $z_{i}$, respectively, represent the actual value and risk threshold of the index.

The composite risk index of these indexes can be calculated by the following function: 
$\mathrm{S}=\sum_{\mathrm{i}=1}^{\mathrm{n}} \mathrm{r}_{\mathrm{i}} * \mathrm{w}_{\mathrm{i}}$

where $S$ is the composite risk index for the sustainable food security and $r_{i}$ and $w_{i}$, respectively, represent the single risk index and the weight of these indexes.

Because the boundary between security and insecurity is vague, this paper uses fuzzy mathematics to classify the grades. The four fuzzy grades are as follows: $\mathrm{N}$ (negligible risk; means the land resources and eco-environmental conditions of this area are very satisfactory and they can fill all the requirements of regional sustainable food security), L (low risk; means the land resources and ecoenvironmental conditions of this area are only moderately, they may insufficient to meet the requirements of regional sustainable food security), M (medium risk; means the land resources and eco-environmental conditions of this area are unsatisfactory, they very likely can't meet the needs of regional sustainable food security) and $\mathrm{H}$ (high risk; means the land resources and eco-environmental conditions of this area are very poor and they certainly can't meet the needs of regional sustainable food security). The corresponding possibility function is shown in Fig. 2, and the membership functions are as follows:
$\mathrm{F}_{\mathrm{N}}(\mathrm{u})=\left\{\begin{array}{c}0 \\ \frac{\mathrm{u}-0.8}{0.1} \\ 1\end{array}\right.$
$0 \leq \mathrm{u} \leq 0.8$
$0.8<\mathrm{u} \leq 0.9$
$0.9<\mathrm{u} \leq 1$
$F_{L}(u)=\left\{\begin{array}{c}0 \\ \frac{u-0.6}{0.1} \\ 1 \\ \frac{0.9-u}{0.1} \\ 1\end{array}\right.$
$0 \leq \mathrm{u} \leq 0.6$
$\mathrm{F}_{\mathrm{M}}(\mathrm{u})=\left\{\begin{array}{c}0 \\ \frac{\mathrm{u}-0.4}{0.1} \\ 1 \\ 0.7-\mathrm{u} \\ \hline 0.1 \\ 1\end{array}\right.$
$0 \leq \mathrm{u} \leq 0.4$
$0.6<\mathrm{u} \leq 0.7$
$0.7<\mathrm{u} \leq 0.8$
$0.8<\mathrm{u} \leq 0.9$
$0.9<\mathrm{u} \leq 1$
$0.4<\mathrm{u} \leq 0.5$
$0.5<u \leq 0.6$
(6) $F_{H}(u)=\left\{\begin{array}{c}1 \\ \frac{0.5-u}{0.3} \\ 0\end{array}\right.$
$0 \leq \mathrm{u} \leq 0.2$
$0.6<\mathrm{u} \leq 0.7$
$0.2<\mathrm{u} \leq 0.5(7)$
$0.7<\mathrm{u} \leq 1$
$0.5<\mathrm{u} \leq 1$

where $u$ represents the single risk index $\left(r_{i}\right)$ or composite risk index $(S), F$ is the possible risk grade of the index.

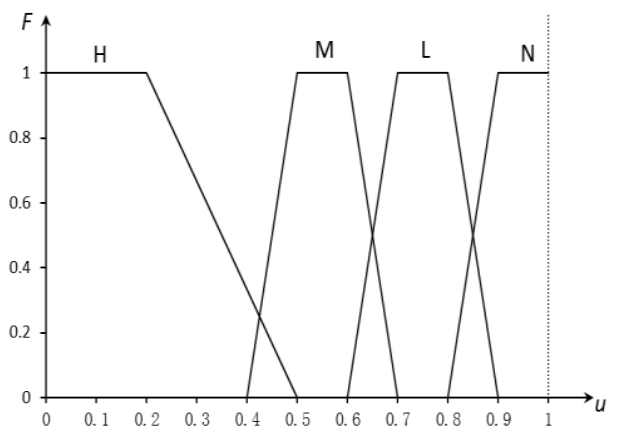

Figure 2. Possibility function of sustainable food security grades

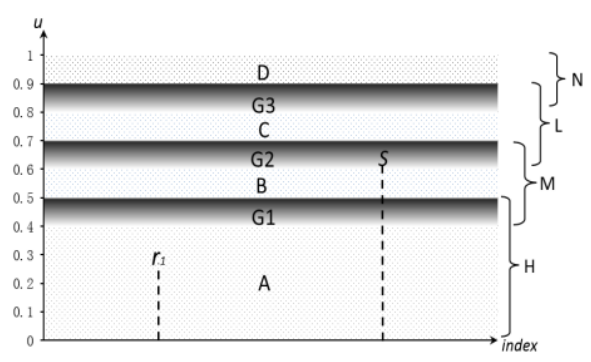

Figure 3. Evaluation series of sustainable food security grades

Based on the possibility function above, the evaluation series can be constructed as shown in Fig. 3. The series are divided into four overlapping areas. They represent high risk (area $A$ and $G 1$ ), medium risk (area G1, B and G2), low risk (area G2, C and G3) and negligible risk (area G3 and D). The corresponding risk grade can be ascertained after calculating the risk index. If the risk index lies in the white area $(A, B, C$ and $D)$, the risk grade can be fixed directly. If the risk index lies in the gray area (G1, G2 and G3), the risk grade needs to be determined according to its two corresponding membership functions (function (4) (7)). 


\subsubsection{Risk evaluation}

Based on the historical data, the single risk indexes and the composite risk indexes over the years can be calculated according to the above functions before building an optimal fitting function using SPSS, which allows the prediction of single risk indexes and composite risk indexes for the next several years. The risk grades can be calculated afterwards.

\section{$3 \quad$ RESULTS}

The six cities (Changsha, Zhuzhou, Xiangtang, Yueyang, Changde and Yiyang) will always be at the medium-risk range due to increasing population pressure, a decrease in the cultivated area, a decrease in the effective irrigation area and agricultural and industrial pollution; however, according to their composite risk indexes, the risk status trends toward getting worse. The number of districts at the medium-risk range, mainly concentrated around the six cities, will increase from six to ten by 2015 .

However, there will be no fundamental changes in risk status in the negligible risk areas in the coming years because of less population pressure, better agricultural infrastructure and better eco-environmental conditions.

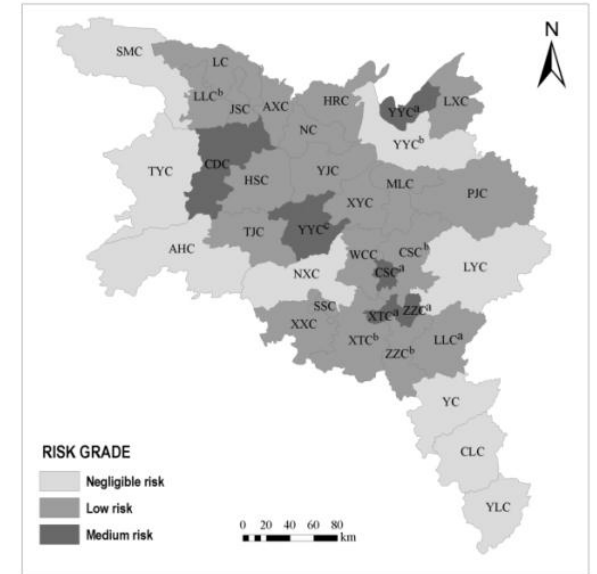

Figure 4. Composite risk ranges in 2010 Figure 5. Composite risk ranges in 2015

\section{DISCUSSION}

Up to now, the policies and measures on food security were always proposed at the national level in china, they cannot be implemented perfectly and reasonably due to diverse eco-environmental conditions and socio-economic conditions in different areas. However, from relevant risk assessment such as this one, the situation could be effectively improved.

Based on the risk assessment for sustainable food security results, with reference to some classical economic methods, using several case studies, an early warning system for sustainable food security could be built. Such a system will be helpful during the risk prevention process for sustainable food security, which will be conducted mainly by the government. 


\section{CONCLUSIONS}

With reference to some classical methods of risk assessment, this paper, using a case study, proposes a process for risk assessment for sustainable food security that focuses on the sustainability of resources and the environment. Its results can provide strong and relevant evidence for agricultural insurance systems and for policy makers in land management departments and the agricultural sector.

Although the current situation is not bad and is not quickly getting worse, throughout, this paper echoes a slight unease because sustainable food security is the basis for sustainable development, especially in major grain-producing areas.

However, because risk assessment for food security is a new area of exploration, there will be some deficiencies in this research, so related studies need to be conducted a more thorough manner.

\section{ACKNOWLEDGMENTS}

This study was supported by the National Natural Science Foundation of China (Project Number 41130526).

\section{REFERENCES:}

Ilbery, B., and D. Maye. Food supply chains and sustainability: Evidence from specialist food producers in the Scottish/English borders .Land Use Policy, 22 (4), 331-344 2005.

Khan, S., and M.A. Hanjra. Footprints of water and energy inputs in food production-Global perspectives. Food Policy, 34(2), 130-140 2009.

Knudsen, I. B. The SAFE FOODS framework for integrated risk analysis of food: An approach designed for science-based, transparent, open and participatory management of food safety. Food Control, 21(12), 1653-1661 2010.

Nie, F.Y., J.Y. Bi, and X.B. Zhang. Study on China's Food Security Status. Agriculture and Agricultural Science Procedia, 1, 301-310 2010.

Shi, X.Q., J.Z. Zhao, and O.Y. ZhiYun. Assessment of eco-security in the Knowledge Grid e-science Environment. The Journal of system and software, 79(2), 246-252 2006.

Smith, M.R., and A. König. Environmental risk assessment for food-related substances. Food Control, 21(12), 1588-1600 2010.

Verbruggen, H.B., and H.J. Zimmermenu. Fuzzy Algorithms for Control. Kluwer Academic Publishers, Boston 1999.

Wu, T.F., H.Q. Deng, and R.T. Wu. Calculation of the Force Structure's Weight Based on PCA. Microcomputer Information, 6(1), 264-265 2009. (in Chinese).

Wu, W.B., P. Yang, and H.J. Tang. A Method for Spatially Explicit Assessment of Potential Risks of Food Insecurity. Agriculture and Agricultural Science Procedia, 1, 325-333 2010.

Yan, H.M., J.Y. Liu, and H.Q. Huang, et al. Assessing the consequence of land use change on agricultural productivity in China. Global and Planetary Change, 67(1-2), 13-19 2009.

Yang, H., and X. B. Li. Cultivated land and food supply in China. Land Use Policy, 17(2), 73-88 2000. 